\title{
AN INTRACTABILITY RESULT FOR MULTIPLE INTEGRATION
}

\author{
I. H. SLOAN AND H. WOŹNIAKOWSKI
}

\begin{abstract}
We prove that the problem of multiple integration in the Korobov class $E_{\alpha, d}$ is intractable since the number of function evaluations required to achieve a worst case error less than 1 is exponential in the dimension.
\end{abstract}

\section{INTRODUCTION}

In this note we consider the approximation of integrals over the $d$-dimensional unit cube,

$$
\text { If }=\int_{[0,1]^{d}} f\left(x_{1}, \ldots, x_{d}\right) d x_{1} \cdots d x_{d}=\int_{[0,1]^{d}} f(x) d x,
$$

under the assumption $f \in E_{\alpha, d}$, where, for arbitrary $\alpha>1, E_{\alpha, d}$ is the set of complex-valued functions in $L_{1}\left([0,1]^{d}\right)$, whose Fourier coefficients satisfy

$$
|\widehat{f}(h)| \leq \frac{1}{\left(\bar{h}_{1} \cdots \bar{h}_{d}\right)^{\alpha}} .
$$

Here $h=\left(h_{1}, h_{2}, \ldots, h_{d}\right)$ with integers $h_{j}$ and

$$
\widehat{f}(h)=\int_{[0,1]^{d}} f(x) e^{-2 \pi i h \cdot x} d x,
$$

$h \cdot x=\sum_{j=1}^{d} h_{j} x_{j}$, and $\bar{h}_{j}=\max \left(1,\left|h_{j}\right|\right)$.

Note that a function $f$ belonging to $E_{\alpha, d}$ necessarily has a continuous 1-periodic extension, since the Fourier series for $f \in E_{\alpha, d}$ is absolutely convergent:

$$
\sum_{h \in \mathbb{Z}^{d}}\left|\widehat{f}(h) e^{2 \pi i h \cdot x}\right| \leq \sum_{h \in \mathbb{Z}^{d}}\left(\bar{h}_{1} \cdots \bar{h}_{d}\right)^{-\alpha}<\infty .
$$

Our aim is to show that in the worst-case setting the integration problem is intractable: that is, to achieve a given error $\varepsilon, \varepsilon<1$, for all $f \in E_{\alpha, d}$, the amount of information required is exponential in $d$. More precisely, we prove that the minimal error of any quadrature rule that uses $N<2^{d}$ points equals 1 . The bound on $N$ is sharp, since, as we shall show, the error of a certain quadrature rule that uses $N=2^{d}$ points may be made arbitrarily small by taking $\alpha$ sufficiently large.

Received by the editor December 21, 1995 and, in revised form, May 3, 1996.

1991 Mathematics Subject Classification. Primary 41A55, 65D30.

Key words and phrases. Multiple integration, intractability, lattice rules.

The first author was partially supported by the Australian Research Council.

The second author was partially supported by the National Science Foundation and the Air Force Office of Scientific Research. 
The class $E_{\alpha, d}$ is standard for the particular class of quadrature formulas known as lattice rules (for a survey see [7]). The implications of the intractability result for lattice methods are considered briefly in Section 3.

Our proof technique is based on an argument which has been already used by Sharygin for a lower bound result for the class $E_{\alpha, d}$, see [6]. An English version of Sharygin's proof can be found in [4], in particular see Theorem 7.17.

\section{The intractability Result}

A quadrature rule approximating (1.1) is a linear functional of the form

$$
Q f=Q(d, N, \omega, t) f:=\sum_{j=1}^{N} \omega_{j} f\left(t_{j}\right),
$$

where the 'weights' $\omega:=\left(\omega_{1}, \ldots, \omega_{N}\right)$ and 'points' $t:=\left(t_{1}, \ldots, t_{N}\right)$ satisfy

$$
\omega_{j} \in \mathbb{C}, \quad t_{j} \in[0,1)^{d}, \quad \text { for } j=1, \ldots, N .
$$

Without loss of generality we can assume that $t_{1}, \ldots, t_{N}$ are distinct points. The worst-case error for the quadrature rule $Q=Q(d, N, \omega, t)$ for the class $E_{\alpha, d}$ is

$$
P_{\alpha}(Q)=P_{\alpha}(d, N, \omega, t):=\sup \left\{|Q f-I f|: f \in E_{\alpha, d}\right\} .
$$

Since we are interested in a lower bound for $P_{\alpha}(Q)$, we define

$$
e(\alpha, d, N):=\inf \left\{P_{\alpha}(d, N, \omega, t): \omega \in \mathbb{C}^{N}, t \in\left([0,1)^{d}\right)^{N}\right\} .
$$

It is an elementary fact that

$$
e(\alpha, d, N) \leq 1,
$$

since by taking $\omega_{1}=\omega_{2}=\cdots=\omega_{N}=0$ we obtain

$$
P_{\alpha}(d, N, 0, t)=\sup \left\{|I f|: f \in E_{\alpha, d}\right\}=\sup \left\{|\widehat{f}(0)|: f \in E_{\alpha, d}\right\}=1 .
$$

The following theorem, which is our main result, states in effect that if $N<2^{d}$ then, in the worst-case setting and for the class $E_{\alpha, d}$, the error is as bad as it can be, and the quadrature rule $Q=0$ is a best possible rule.

Theorem 1. If $N<2^{d}$, then

$$
e(\alpha, N, d)=1 .
$$

Proof. Let $N<2^{d}$, and suppose that points $t=\left(t_{1}, \ldots, t_{N}\right)$ and weights $\omega=$ $\left(\omega_{1}, \ldots, \omega_{N}\right)$ are given. The theorem is proved by constructing a function $g \in E_{\alpha, d}$, depending on $t$ and $\omega$, such that $I g=1$ and $Q g=0$. From this it will follow that $P_{\alpha}(Q) \geq|Q g-I g|=|I g|=1$. Since this holds for any choice of points $t$ and weights $\omega$, it follows that $e(\alpha, N, d) \geq 1$, which together with $(2.1)$ proves $e(\alpha, N, d)=1$.

To accomplish the construction, let $B_{d}:=\{0,1\}^{d}$, and define $g$ to be a trigonometric polynomial of the form

$$
g(x)=\theta(x) \sum_{h \in B_{d}} a_{h} e^{2 \pi i h \cdot x},
$$

where $\left\{a_{h} \in \mathbb{C}: h \in B_{d}\right\}$ is a non-trivial solution of the linear system

$$
\sum_{h \in B_{d}} a_{h} e^{2 \pi i h \cdot t_{j}}=0, \quad j=1, \ldots, N
$$

and $\theta$ is a trigonometric polynomial which is yet to be specified. A crucial point in the proof is that, because the homogeneous linear system (2.3) has $2^{d}$ unknowns 
but fewer than $2^{d}$ equations, a non-trivial solution of (2.3) certainly exists. Let $h^{*} \in B_{d}$ be such that $\left|a_{h}\right| \leq\left|a_{h^{*}}\right|$ for $h \in B_{d}$. We scale our non-trivial solution of (2.3) so that

$$
\left|a_{h}\right| \leq 1 \quad \text { for } \quad h \in B_{d} \quad \text { and } \quad a_{h^{*}}=1 .
$$

It follows from (2.2) and (2.3) that

$$
g\left(t_{j}\right)=\theta\left(t_{j}\right) \sum_{h \in B_{d}} a_{h} e^{2 \pi i h \cdot t_{j}}=0, \quad j=1, \ldots, N,
$$

from which it is clear that $Q(d, N, \omega, t) g=0$. Now we choose

$$
\theta(x):=e^{-2 \pi i h^{*} \cdot x},
$$

so that

$$
g(x)=\sum_{h \in B_{d}} a_{h} e^{2 \pi i\left(h-h^{*}\right) \cdot x} .
$$

Clearly

$$
I g=\widehat{g}(0)=a_{h^{*}}=1 .
$$

On the other hand $g$ given by (2.4) is a trigonometric polynomial of degree $\leq 1$ in each component of $x$, since for $h, h^{*} \in B_{d}$ we have

$$
h_{j}-h_{j}^{*}=0,1 \text { or }-1 \text { for } j=1, \ldots, d .
$$

This implies

$$
\overline{\left(h_{1}-h_{1}^{*}\right)} \overline{\left(h_{2}-h_{2}^{*}\right)} \cdots \overline{\left(h_{d}-h_{d}^{*}\right)}=1 \text { for } h, h^{*} \in B_{d} .
$$

It therefore follows, since $\left|a_{h}\right| \leq 1$, that $g \in E_{\alpha, d}$, and so the theorem is proved.

Remark 1. Theorem 1 remains valid if we permit more general quadrature rules. Namely, for fixed points $t_{j}$ we may approximate the integral If by $\phi\left(f\left(t_{1}\right), \ldots, f\left(t_{n}\right)\right)$, where $\phi$ is an arbitrary nonlinear mapping, $\phi: \mathbb{R}^{n} \rightarrow \mathbb{R}$. Since the class $E_{\alpha, d}$ is convex and symmetric (i.e., $f \in E_{\alpha, d}$ implies that $-f \in E_{\alpha, d}$ ) we may apply Smolyak's theorem, see e.g., [9] p. 76. This theorem states that the mapping $\phi$ which minimizes the worst-case error is linear. For linear $\phi$ we have just proven that the error is at least one.

We may also permit adaptive choice of points $t_{j}$. That is, assuming that the points $t_{1}, \ldots, t_{j-1}$ are already chosen and the function values $f\left(t_{1}\right), \ldots, f\left(t_{j-1}\right)$ are already computed, the next point $t_{j}$ may depend arbitrarily on $f\left(t_{1}\right), \ldots, f\left(t_{j-1}\right)$. By Bakhvalov's theorem, see e.g., [9], p. 59, the worst-case error of arbitrary quadrature rule that uses adaptive points $t_{j}$ cannot be smaller than the minimal worst-case error of linear quadrature rules that use non-adaptive (fixed) points. Again the latter error is at least 1 . Hence, Theorem 1 also holds for adaptive choice of points $t_{j}$.

\section{LATTICE RULE RESUlts}

Our purpose in this section is merely to mention some known lattice rule results that cast an interesting light on the result in Theorem 1. In particular, we will see that the condition $N<2^{d}$ in the theorem cannot be improved, and indeed that the behaviour of $e(\alpha, N, d)$ changes dramatically when $N$ reaches $2^{d}$. 
A lattice rule is an equal-weight rule of the form

$$
Q f=\frac{1}{N} \sum_{j=1}^{N} f\left(t_{j}\right)
$$

where

$$
\left\{t_{1}, \ldots, t_{N}\right\}=L \cap[0,1)^{d},
$$

and $L$ is an 'integration lattice'; that is to say, $L$ is a geometrical lattice containing $\mathbb{Z}^{d}$ as a subset, where a geometrical lattice is a discrete subset of $\mathbb{R}^{d}$ that is closed under addition and subtraction. It is known [8] that if $Q$ is a lattice rule that corresponds to an integration lattice $L$, then

$$
Q f-I f=\sum_{\substack{h \in L^{\perp}, h \neq 0}} \widehat{f}(h) \quad \text { for } \quad f \in E_{\alpha, d},
$$

where $L^{\perp}$ is the 'reciprocal lattice' of $L$,

$$
L^{\perp}:=\left\{h \in \mathbb{R}^{d}: x \cdot h \in \mathbb{Z}, \forall x \in L\right\} \subseteq \mathbb{Z}^{d} .
$$

It follows in turn that

$$
P_{\alpha}(Q):=\sup \left\{|Q f-I f|: f \in E_{\alpha, d}\right\}=\sum_{\substack{h \in L^{\perp}, h \neq 0}} \frac{1}{\left(\overline{h_{1}} \cdots \overline{h_{d}}\right)^{\alpha}} .
$$

One of the simplest of all lattice rules is the $n^{d}$-point product-rectangle rule

$$
R_{n} f:=\frac{1}{n^{d}} \sum_{k_{1}=0}^{n-1} \sum_{k_{2}=0}^{n-1} \ldots \sum_{k_{d}=0}^{n-1} f\left(\frac{k_{1}}{n}, \frac{k_{2}}{n}, \ldots, \frac{k_{d}}{n}\right) .
$$

For this rule we can easily compute $P_{\alpha}\left(R_{n}\right)$, by using the fact that the corresponding integration lattice is $L=\left(n^{-1} \mathbb{Z}\right)^{d}$, from which it follows that $L^{\perp}=(n \mathbb{Z})^{d}$. Specifically, from (3.1) we find

$$
P_{\alpha}\left(R_{n}\right)=\prod_{j=1}^{d}\left(\sum_{h_{j} \in n \mathbb{Z}} \frac{1}{{\overline{h_{j}}}^{\alpha}}\right)-1=\left(1+\frac{2 \zeta(\alpha)}{n^{\alpha}}\right)^{d}-1,
$$

where $\zeta(x)$ is the Riemann zeta function,

$$
\zeta(x)=\sum_{i=1}^{\infty} i^{-x}, \quad \text { for } \quad x>1 .
$$

In particular, on setting $n=2$ we find

$$
P_{\alpha}\left(R_{2}\right)=\left(1+\zeta(\alpha) / 2^{\alpha-1}\right)^{d}-1 .
$$

In the product-rectangle rule with $n=2$ we have $N=2^{d}$, thus this example only just misses being covered by Theorem 1. On the other hand, we note from (3.3) that

from which it follows that

$$
P_{\alpha}\left(R_{2}\right) \longrightarrow 0 \quad \text { as } \quad \alpha \longrightarrow \infty,
$$

$$
e\left(\alpha, 2^{d}, d\right) \longrightarrow 0 \quad \text { as } \quad \alpha \longrightarrow \infty .
$$

And since $e(\alpha, N, d)$ is clearly non-increasing in $N$, it follows in turn that

$$
e(\alpha, N, d) \longrightarrow 0 \quad \text { as } \quad \alpha \longrightarrow \infty \text { for all } N \geq 2^{d} \text {. }
$$


The last result stands in striking contrast to the result from the theorem that

$$
e(\alpha, N, d)=1 \quad \forall \alpha>1 \quad \text { if } \quad N<2^{d} .
$$

This result (3.4) shows that the condition $N<2^{d}$ in the theorem cannot be weakened, at least for large values of $\alpha$.

The product-rectangle rule is not usually thought of as an interesting lattice rule, because lattice rules are traditionally designed to have good asymptotic convergence properties as $N \longrightarrow \infty$ (for fixed $d$.) By this test the $n^{d}$-point product-rectangle rule performs poorly, since (3.2) gives the inferior asymptotic result

$$
P_{\alpha}\left(R_{n}\right)=O\left(n^{-\alpha}\right)=O\left(N^{-\alpha / d}\right) .
$$

Much greater interest attaches usually to the 'method of good lattice points', a class of lattice rules of the form

$$
Q(z) f:=\sum_{j=0}^{N-1} f\left(\left\{j \frac{z}{N}\right\}\right),
$$

where $z \in \mathbb{Z}^{d}$ is a well chosen integer vector, with no nontrivial factor in common with $N$, and $\{x\}$ for $x \in \mathbb{R}^{d}$ means that each component of $x$ is to be replaced by its fractional part in $[0,1)$.

The classical theorems of the method of good lattice points (see, for example, [5] or [7]) assert the existence of $z=z(N)$ such that

$$
P_{\alpha}(Q(z)) \leq c(\alpha, d) \frac{(\log N)^{\beta(\alpha, d)}}{N^{\alpha}},
$$

for some positive functions $c$ and $\beta$. Usually, $\beta(\alpha, d)$ is of order $d$. This result indicates an impressive rate of convergence for large $N$, but asymptotic bounds of this kind either do not give explicit values of $c(\alpha, d)$, or do not provide useful bounds for smaller values of $N$. Among the known explicit bounds, the authors of [2] assert that for prime values of $N$ up to approximately $10^{d}$ the bound in the following theorem is as good as any known bound:

Theorem 2. For $N$ prime there exists a lattice rule $Q(z)$ of the form (3.5) such that

$$
P_{\alpha}(Q(z)) \leq M_{\alpha}=\frac{(1+2 \zeta(\alpha))^{d}}{N}+\frac{N-1}{N}\left(1-\frac{2\left(1-N^{1-\alpha}\right) \zeta(\alpha)}{N-1}\right)^{d}-1 .
$$

Here $M_{\alpha}$ is just the mean of $P_{\alpha}\left(Q\left(z^{\prime}\right)\right)$ over all integer vectors $z^{\prime}$ with components $z_{j}^{\prime}$ satisfying $-N / 2<z_{j}^{\prime} \leq N / 2, \quad z_{j}^{\prime} \neq 0$.

An analogous result for composite $N$ is given in [1].

The following proposition shows that if $N \approx 2^{d}$ and $N \geq 2 \zeta(\alpha)+1$, then the bound in Theorem 2 for the method of good lattice points is larger (and hence less good) than $P_{\alpha}$ for the humble $2^{d}$-point product-rectangle rule, given by (3.3).

Proposition 1. If $N=2^{d} \geq 2 \zeta(\alpha)+1$, then

$$
\frac{(1+2 \zeta(\alpha))^{d}}{N}+\frac{N-1}{N}\left(1-\frac{2\left(1-N^{1-\alpha}\right) \zeta(\alpha)}{N-1}\right)^{d}-1 \geq\left(1+\frac{\zeta(\alpha)}{2^{\alpha-1}}\right)^{d}-1 .
$$


Proof. Because $N \geq 2 \zeta(\alpha)+1$, we see that

$$
1-\frac{2\left(1-N^{1-\alpha}\right) \zeta(\alpha)}{N-1} \geq 1-\frac{2 \zeta(\alpha)}{N-1} \geq 0,
$$

from which follows

$$
\begin{gathered}
\frac{(1+2 \zeta(\alpha))^{d}}{N}+\frac{N-1}{N}\left(1-\frac{2\left(1-N^{1-\alpha}\right) \zeta(\alpha)}{N-1}\right)^{d}-1 \\
\geq \frac{(1+2 \zeta(\alpha))^{d}}{N}-1=\left(\frac{1}{2}+\zeta(\alpha)\right)^{d}-1,
\end{gathered}
$$

since $N=2^{d}$. The result now follows from the inequality

$$
\frac{1}{2}+\zeta(\alpha) \geq 1+\frac{\zeta(\alpha)}{2^{\alpha-1}},
$$

which is trivial for $\alpha \geq 2$, and proved for all $\alpha>1$ in Proposition 4, Part (i) of [3].

The fact that $P_{\alpha}$ for the $2^{d}$-point product-rectangle rule is smaller than $M_{\alpha}$, which is the mean value of $P_{\alpha}$ for prime-order rules of the form (3.5), is an echo of a result proved in [3] for $2^{d} k$-point rules which are ' $2^{d}$-copies' of $k$-point rules of the form (3.5) with $k$ prime. There it is shown that the mean $P_{\alpha}$ for such $2^{d} k$-point copy rules is smaller than the mean $M_{\alpha}$ for rules of the form (3.5) with $N \approx 2^{d} k$. The $2^{d}$-point product-rectangle rule is the $2^{d}$-copy of the 1 -point rule $Q f=f(0)$, thus Proposition 1 in effect extends the result in [3] on the merit of $2^{d}$-copy rules to the case $k=1$.

\section{REFERENCES}

[1] Disney, S.A.R. Error bounds for rank 1 lattice quadrature rules modulo composites. Monatshefte für Mathematik, 110, 89-100 (1990). MR 91m:65067

[2] Disney, S.A.R. and Sloan, I.H. Error bounds for the method of good lattice points. Mathematics of Computation, 56, 257-66 (1991). MR 91m:65068

[3] Disney, S.A.R. and Sloan, I.H., Lattice integration rules of maximal rank formed by copying rank 1 rules, SIAM Journal on Numerical Analysis, 29, 566-77 (1992). MR 92k:65034

[4] Hua, L. K. and Wang, Y., Applications of number theory to numerical analysis. Springer Verlag, Berlin (1981). MR 83g:10034

[5] Niederreiter, H. Random number generation and quasi-Monte Carlo methods. Society for Industrial and Applied Mathematics, Philadelphia (1992). MR 93h:65008

[6] Sharygin, I. F., A lower estimate for the error of quadrature formulas for certain classes of functions (in Russian), Zh. Vychisl. Mat. i Mat. Fiz., 3, 370-376 (1963).

[7] Sloan, I.H. and Joe, S. Lattice methods for multiple integration. Oxford University Press, Oxford (1994).

[8] Sloan, I.H. and Kachoyan, P.J. Lattice methods for multiple integration: theory, error analysis and examples. SIAM Journal on Numerical Analysis, 24, 116-28 (1987). MR 88e: 65023

[9] Traub, J.F., Wasilkowski, G. and Woźniakowski, H. Information-based complexity. Academic Press, Boston (1988). MR 90f:68085

School of Mathematics, University of New South Wales, Sydney 2052, Australia

Department of Computer Science, Columbia University, New York, New York 10027 and Institute of Applied Mathematics, University of WarsaW, ul. Banacha 2, 02-097 WarsaW, Poland 